\title{
EL RESIDUO INVISIBLE. REALIDAD, IDEALIDAD Y FENOMENOLOGÍA DEL TIEMPO EN LA FILOSOFÍA CRÍTICA KANTIANA
}

\section{Angel Garrido Maturano*}

"Hay interminables objeciones contra la arbitrariedad de mis interpretaciones. La presente obra puede muy bien servir de base a tales objeciones. En efecto, los historiadores de la filosofía tienen razón cuando enfocan su crítica contra quienes tratan de exponer un diálogo de pensamientos entre pensadores. Pues un diálogo deesta clase, a diferencia de los métodos propios de la filosofia bistórica, se halla bajo muy diversas leyes. Son leyes más vulnerables. En los diálogos el peligro de errar es mayor, los defectos más frecuentes. (...). Quienes piensan, aprenderán más a partir de las deficiencias". Martin, Heidegger, prólogo a la segunda edición de Kant y el problema de la metafísica.

\section{Introducción}

Este artículo se ocupa de un tema tratado hasta el hartazgo por la investigación filosófica: la cuestión del tiempo en Kant. ¿Era necesario fatigar al esforzado lector con un nuevo trabajo sobre un tema que ha sido ya minuciosa y puntualmente indagado? En cierto sentido no. Por eso renuncio conscientemente a una nueva exposición detallada y erudita de la doctrina kantiana sobre el tiempo; por eso desisto de toda reconstrucción de la genealogía y desarrollo de la comprensión de Kant del fenómeno; por eso, en suma, prescindo de cualquier intento de realizar aquí un "comentario crítico" que examine la función que cumple la doctrina del tiempo en la arquitectura y problemática de la filosofía kantiana ${ }^{1}$. Pero en cierto sentido sí, en cuanto la

\footnotetext{
* Investigador del CONICET, Profesor de seminario de posgrado en el Doctorado en Filosofía de la UCSF, docente Fac. de Humanidades de la Univ. Nacional del Nordeste, Resistencia (hieloypuna@hotmail.com).

1 Para hacer un inútil alarde de erudición se podría confeccionar una larga lista de libros y artículos que asumen la perspectiva que este trabajo esquiva. Quiero, empero, mencionar sólo tres obras clásicas que son accesibles en español y que, hasta donde mi modesto conocimiento bibliográfico alcanza, siguen siendo, por su rigurosidad, sistematicidad y completud, indicadas para que los hispanohablantes
} 
perspectiva bermenéutica desde la que pretendo abordar la cuestión, si bien no es nueva, puede, a mi parecer, ser fecunda y dar pie a una reflexión filosófica fundamental sobre el origen y los límites de toda meditación sobre el tiempo. ¿Cuál es, pues, esta perspectiva? Se trata de un análisis que se centra en examinar los fundamentos de la doctrina del tiempo en Kant en el marco de la primera de las tres aporías de la temporalidad que Paul Ricoeur estudia en Tiempo y narración $I I I^{2}$. Esta aporía podría denominarse "aporía de la ocultación mutua de las dos perspectivas sobre el tiempo" y concretamente mienta la imposibilidad de reducir el tiempo real o cósmico (el tiempo señalado por el curso de los astros) al tiempo ideal o fenomenológico (el tiempo como experiencia del propio decurso de la conciencia), y visceversa. Tal irreductibilidad resulta de una diferencia esencial entre ambas formas del tiempo. Por una parte, el tiempo cósmico es caracterizable como una continuidad de momentos homogéneos cualesquiera, en la cual no se puede distinguir la dimensión del pasado, ni la del presente, ni la del futuro, sino tan sólo el continuo transformarse del universo. Por otra parte, el tiempo fenomenológico se puede caracterizar como el despliegue ex-tático de los horizontes de pasado y de futuro a partir del ahora autorreferencial de la conciencia, el cual ya no es un momento cualquiera, sino el presente viviente en el que la conciencia experimenta su propio decurso. En resumidas cuentas se trata de una doble diferencia: cualitativa, entre un tiempo sin presente y otro con presente; y cuantitativa, entre un tiempo determinado, que comienza en un presente definido y finaliza con la muerte, y otro indeterminado, cuyos límites exceden los de la conciencia individual y nos son ignotos. Pero, ¿por qué abordar la cuestión del tiempo en Kant en el

estudiemos no sólo la cuestión del tiempo en Kant, sino el conjunto del pensamiento kantiano. Ellas son: Gottfried Martin, Kant. Ontologia y epistemologia, trad. De L. F. Carrer y A. Raggio, Universidad Nacional de Córdoba, Córdoba, 1961; Herman Jean de Vleeschauwer, La evolución del pensamiento kantiano. Historia de una doctrina, México, 1962; y la obra siempre vigente de Roberto Torreti, Kant, Buenos Aires, Charcas, 1980. Para profundizar este estudio se podrían señalar otras dos obras de dos de los tres autores citados: Gotfried Martin, Gesammelte Abbandlungen Bd. 1, Köln, 1961 y Herman Jean de Vleeschauwer, La déduction trascendentale dans l'oeuvre de Kant (3 vols), Paris/Lenoux/S'Gravenhage, M. Nijhoff, 1934-1937.

2 Cf. Paul Ricoeur, Tiempo y narración III. El tiempo narrado, trad. A. Neira, Madrid/México, Siglo XXI, en especial pp. 694-717. De aquí en más esta obra se cita de acuerdo con la siguiente sigla TN III. 
marco de esta aporía? Porque parto de la suposición de que el análisis kantiano del tiempo (en particular el desarrollado en la Crítica de la razón pura ${ }^{3}$, obra a la que se restringe esta investigación) muestra que la consideración ideal del tiempo como una forma subjetiva de la intuición y la visión real del fenómeno como una magnitud objetiva del universo no resultan opuestas e inconciliables, sino, por el contrario, se trata de perspectivas que se entrecruzan, conviven y que necesariamente se requieren la una a la otra.

De acuerdo con lo dicho, los objetivos centrales de este trabajo serán tres. En primer lugar aspiro a mostrar en qué medida la determinación ideal del tiempo en la estética trascendental como forma a priori de la intuición no implica en modo alguno que la concepción kantiana del tiempo no se encuentre referida a un tiempo real dado, a saber, "el tiempo que en sí mismo no puede ser percibido". En segundo lugar, y en estricta relación con el objetivo anterior, buscaré poner a la luz en qué medida la determinación objetiva del tiempo como tiempo de la naturaleza, desplegada por Kant sobre todo en las analogías de la experiencia, supone tanto el tiempo real cuanto una fenomenología encubierta del tiempo. Dicho con mayor precisión: procuraré mostrar, en primer lugar, en qué medida el tiempo real, cósmico o tiempo propiamente dicho, aun cuando no pueda ser asido en sí mismo, debe suponerse como determinante de las características generales de la temporalidad propia de las relaciones objetivas entre los fenómenos; y, en segundo lugar, en qué medida esta temporalidad implica a su vez una fenomenología encubierta de la temporalidad conciencial inmanente. Finalmente, y a modo de corolario que excede los marcos de un artículo estrictamente dedicado a Kant, intentaré indicar brevemente el sentido, esto es, aquello sobre la base de lo cual es posible que ambas perspectivas de análisis del tiempo, la fenomenológica o subjetiva y la objetiva o cósmica, se

${ }^{3} \mathrm{La}$ Crítica de la Razón Pura se cita de aquí en adelante según la traducción de la siguiente edición en español: Inmanuel Kant, Crítica de la Razón Pura, trad. M. García Morente y M. Fernández Núñez, México, Ed. Porrúa, 1982. Sigla: CRP. Además, en todos los casos se consigna entre paréntesis la ubicación del pasaje citado según la paginación original. Para el texto original alemán nos hemos regido por la siguiente edición: Inmanuel Kant, Sämtliche Werke, Hrsg. K. Vorländer: erster Band Kritik der reinen Vernunft, nach der ersten und zweiten Originalausgabe herausgegeben von Raymund Schmidt, Leipzig, Felix Meiner, 1926. Sigla: KrV. 
imbriquen mutuamente y cómo esa imbricación mutua representa una frontera insalvable con la que se topa toda investigación sobre el tiempo.

Desde el punto de vista del desarrollo temático comenzaré examinando la estética trascendental. A continuación me referiré a las analogías de la experiencia. Finalmente haré hincapié en la refutación kantiana del idealismo. Desde el punto de vista del método quizás no sea ocioso aclarar que en cada caso nuestro examen de estos temas clásicos del pensamiento kantiano implicará su reinterpretación. Esta reinterpretación quiere cumplirse poniendo en diálogo los textos de Kant con dos de sus lecturas filosóficas más fecundas: la de Ricoeur y la de Heidegger ${ }^{4}$.

\section{La estética trascendental: la idealidad real del tiempo}

Kant comienza su análisis del tiempo y del espacio afirmando que son intuiciones a priori y que pertenecen, por tanto, al orden de la sensibilidad definida como "la capacidad (receptividad) de recibir representaciones por el modo como somos afectados por objetos" ¿Cómo entender la paradoja de una intuición a priori, es decir, que no depende de la afección de nuestra sensibilidad por ningún objeto externo? Según Kant, esto sólo es posible si se trata de una representación de la propia sensibilidad, es decir, de una autoafección de la sensibilidad, que, autoafectándose, conforma las condiciones espacio-temporales de acuerdo con las cuales ha de recibir toda otra afección de objetos externos. Ya en este punto se podría preguntar por qué la sensibilidad se autoafecta espacio- temporalmente y no puede no bacerlo. El que no pueda no bacerlo es indicador de un dato tan paradojal como relevante para nuestro análisis: la sensibilidad es respecto de sus propias representaciones activa y pasiva. Activa porque tiempo y espacio son representaciones suyas a priori que no dependen de los datos de los sentidos; y pasiva porque son representaciones que ella no puede no representar. En

${ }^{4} \mathrm{El}$ diálogo con Ricoeur es explícito y, en general, mi visión se acerca a la del filósofo francés, aun cuando pretendo acentuar más fuertemente las razones de por qué suponer una dimensión real del tiempo en Kant. En cambio el diálogo con la interpretación de Heidegger, que coloca la temporalidad del sí mismo como raíz última del tiempo, permanece casi siempre soterrado e implícito y, no habré de negarlo, es de tono polémico.

${ }^{5} \mathrm{CRP}$, p. 41; KrV A 19/ B 33. 
otros términos a la sensibilidad le está dado el tener que representarse espacio y tiempo. Y como el tiempo es la forma intuitiva a priori de todos los fenómenos ( $\mathrm{y}$ no sólo de los fenómenos externos, sino también de los propios estados de conciencia) podemos decir que la sensibilidad pre-supone en cualquier representación suya su propia representación intuitiva y a priori del tiempo. El tiempo no es, pues, estrictamente hablando, un producto de la sensibilidad, sino que para la sensibilidad es un dato tener que intuir a priori temporalmente sus objetos. Por lo cual cabe preguntarse si la sensibilidad no presupone un tiempo real dado que no puede sino intuir en cada una de sus representaciones. De este modo el tiempo sería a la vez ideal (un fenómeno) en cuanto nada sabemos de cómo sea el tiempo fuera de nuestra intuición $a$ priori de él como condición formal de toda representación de objetos. Pero, a la par, el tiempo sería real en cuanto es un dato de la realidad que tengamos que presuponer que hay tiempo, porque la sensibilidad no puede no producir sus representaciones si no es en el tiempo. Éste es el punto de partida de mi interpretación de la estética.

En ella, como es sabido, Kant realiza las exposiciones metafísica y trascendental del tiempo. En la exposición trascendental se explica cómo una representación pura de la sensibilidad constituye un principio a partir del cual son posibles conocimientos sintéticos a priori, tantos como ofrece la teoría general del movimiento ${ }^{6}$ (la mecánica). En otros términos, del hecho de que necesariamente intuimos el tiempo en una cierta forma, se derivan conocimientos necesarios y universales sobre los procesos temporales que no exigen una experiencia previa de las realidades empíricas a las cuales se aplican. La exposición trascendental en esencia afirma el carácter apodíctico y universal de la unidimensionalidad u homogeneidad lineal del tiempo: "diversos tiempos no son a la vez, sino unos tras otros"? Sobre la base de esta apodicticidad, que no puede provenir de las sensaciones empíricas cambiantes, se fundan los principios sintéticos a priori que regulan las relaciones temporales entre todos los fenómenos que constituyen la experiencia científica. Aquí cabría preguntarse ¿de dónde le viene a la sensibilidad esta certeza apodíctica de la unidimensionalidad del tiempo? Una respuesta posible, que no responde demasiado, es: "porque la sensibilidad está constituida así”. Otra, aquella que prefiero, nos dice que la sensibilidad

\footnotetext{
${ }^{6} \mathrm{Cf} . \mathrm{KrV}$, B 49.

${ }^{7} \mathrm{CRP}$, p. $47 ; \mathrm{KrV}$, A $31, \mathrm{~B} 47$.
} 
no puede estar constituida de otro modo porque ella en cada una de las percepciones de los fenómenos está referida a un tiempo unidimensional que regula el modo en que experimenta las relaciones temporales entre ellos. Veremos luego en detalle que esta interpretación se compatibiliza con la concepción kantiana de las analogías de la experiencia, pero digamos aquí a modo de adelanto que si la unidimensionalidad del tiempo fuese sólo resultante de la constitución subjetiva de un espíritu finito, entonces habría que concluir, por ejemplo, que el principio de conservación de la sustancia (diríamos hoy de la energía), que supone un tiempo infinito y unidimensional en el cual los fenómenos se transforman sucesivamente manteniéndose un quantum energético, no sería una constante del cosmos, sino tan sólo un modo en que el hombre intuye el cosmos. Ahora bien, el principio vale antes y después de la existencia humana, por lo que me resulta muy difícil explicarlo, si se supone que esa unidimensionalidad del tiempo que requiere como su condición de posibilidad no remite a una dimensión real (aunque inasible en sî) del fenómeno, sino tan sólo a la constitución de un espíritu finito.

En la exposición metafísica se despliegan una serie de conocidos argumentos destinados a probar por vía refutatoria los dos primeros el carácter a priori y los dos últimos el carácter intuitivo del tiempo. El primer argumento afirma que el tiempo no es un concepto empírico porque la simultaneidad o la sucesión no se extraen por abstracción de diversas percepciones de objetos empíricos, sino que estas percepciones las presuponen, es decir, ya siempre son simultáneas o sucesivas. El segundo demuestra el carácter a priori del tiempo afirmando que no es posible representarse objetos que no estén en el tiempo, pero si un tiempo libre de objetos. No vamos a discutir aquí en detalle este argumento, que merecería múltiples objeciones ${ }^{8}$, sin embargo tampoco podemos dejar de referirnos un poco más in extenso a él, puesto que, a primera vista, cuestiona nuestra hipótesis de la existencia de un tiempo real presupuesto por la intuición a priori del tiempo. En efecto, si la sensibilidad pudiese representarse un tiempo vacío, un tiempo en sí, independiente de cualquier experiencia de objetos en el tiempo, se podría colegir de ello que el tiempo mismo es un producto de la sensibilidad. No habría que presuponer, como lo hemos hecho más arriba, que haya un tiempo real, ajeno a toda intuición de objetos,

8 Para tal discusión cf. R. Torreti, op. cit., pp. 180-183. 
pero que sólo es asido indirectamente por la sensibilidad a través de su intuición de relaciones de objetos en el tiempo, sino que este propio tiempo podría ser producido e intuido en sí mismo por la imaginación. Ahora bien, ¿de dónde saca Kant que se puede percibir el tiempo puro? Es imposible imaginarse un tiempo absolutamente vació en el que no ocurra nada, porque en tal caso no habría referencia para hablar de permanencia, simultaneidad o sucesión, que son las formas más generales del tiempo. Pero, además y por sobre todo, es imposible imaginarse un tiempo tan vacío en que ni siquiera se distinga en él el propio estado de conciencia del sujeto que está experimentando ese tiempo vacío. En efecto, toda experiencia del tiempo presupone por lo menos una autoexperiencia de la conciencia que experimenta su propio decurso en ese tiempo presuntamente vacío. Decimos "presuntamente", porque un tiempo absolutamente vacío es un tiempo absolutamente homogéneo e indistinto, pero en cuanto suponemos que podemos imaginarlo de tal modo, introducimos el ahora en que lo estamos imaginando, y ese ahora sirve de punto de referencia distinto de todos los otros instantes vacíos. En conclusión, desde el punto de vista gnoseológico el tiempo, a pesar de ser a priori, de no ser sacado por abstracción de las diversas percepciones de objetos empíricos como establece el primer argumento, nunca es experimentado por sí mismo (cosa que el propio Kant, como veremos en nuestro tratamiento de las analogías, una y otra vez repite $^{9}$ ). Y, desde el punto de vista ontológico, tampoco puede ser concebido el tiempo como tiempo efectivo, si en él nada pasa, nada dura ni nada se transforma. En efecto, ¿qué sería un tiempo vacío, un tiempo en él que no se pudiesen establecer relaciones entre las cosas en función de la constitución de nuestra experiencia? Sería una especie de super-recipiente que subsiste siempre idéntico a sí mismo, pero con la peculiaridad de no contener nada dentro ni tampoco contornos que lo definan. Con lo cual ni siquiera podría decirse que es un recipiente. Sería la mera subsistencia de la nada. Hay, pues, que renunciar a esta idea de un tiempo percibido por sí mismo, e interpretar el argumento kantiano no en términos gnoseológicos ni ontológicos, sino fenomenológicos y afirmar que el tiempo, considerado independientemente de todas las transformaciones o movimientos a través de los cuales el sujeto intuye su pasar, no es más que la proyección (o protensión) y retroyección (o retención) de un horizonte temporal a partir del ahora en que la conciencia

${ }^{9}$ Por ejemplo $C R P$, p. 116, $K r V$, B 219. 
experimenta (no un tiempo vacío) sino a sí misma experimentando o intuyendo un objeto en el tiempo. Por lo tanto, ese tiempo sin objetos no puede mentar un tiempo vacío absolutamente homogéneo, sino que en él ya están introducidas las dimensiones de presente, pasado y futuro. Cómo conclusión de este segundo argumento habrá que hacer notar, pues, la incapacidad de la sensibilidad para producir o intuir el tiempo puro, absolutamente indiferenciado y lineal, y su necesidad de suponer que "hay tiempo" puesto que, como dijimos antes, el sujeto sólo puede percibir las relaciones entre los fenómenos como relaciones temporales, y, agregamos ahora, sólo puede ser autoconsciente de modo temporal. Hay, pues, aquí una especie de paradoja insoluble: no hay tiempo sin la intuición subjetiva de algo en el tiempo, a saber, las transformaciones fenoménicas o la transformación de los propios estados de conciencia siempre en relación con las transformaciones fenoménicas como su punto de referencia. Pero la intuición no produce esta transformación ni puede detenerla, por lo que ella remite a un tiempo real que la sobrepasa, pero que en sí mismo es inasible. $\mathrm{El}$ tercer argumento defiende el carácter intuitivo del tiempo y niega que éste sea un universal genérico. Se basa en el hecho de que no extraemos el concepto del tiempo reuniendo en un concepto genérico las notas comunes de diferentes tiempos, sino que los diferentes tiempos constituyen lapsos sucesivos dentro de un mismo tiempo. El tiempo constituye, por tanto, un objeto único y "la representación que no puede ser dada más que por un objeto único, es intuición"10. El cuarto argumento, que también defiende el carácter intuitivo del tiempo en tanto objeto único, afirma que nuestra representación originaria del tiempo es ilimitada, es decir, infinita, y "la infinitud del tiempo no significa otra cosa que toda magnitud determinada del tiempo es sólo posible mediante limitaciones de un tiempo único fundamental (einer einzigen zum Grunde liegenden Zeit)"11. Por tanto, el tiempo fundamental, del cual las distintas magnitudes temporales representan lapsos limitados, no puede ser un concepto, sino el objeto único de una intuición inmediata. En efecto, aun cuando un concepto genérico es una representación que está contenida en una multitud potencialmente infinita de representaciones particulares, como rasgo común presente en todas ellas, sin embargo ningún concepto contiene una multitud infinita de representaciones.

\footnotetext{
${ }^{10} \mathrm{CRP}$, p. 47; KrV, A 32, B 47.

${ }^{11} \mathrm{CRP}$, pp. 47-48; $\mathrm{KrV}$, A 32, B 48.
} 
Pero el tiempo contiene infinitos lapsos temporales como lapsos suyos, lapsos de un único tiempo, por lo tanto, el tiempo no puede ser un concepto, sino una intuición a priori. Ahora bien, ¿cómo puede ser intuible una magnitud infinita? Ciertamente esta pregunta, como señala Torreti ${ }^{12}$, sólo puede ser respondida si se supone la conciencia de poder hacer progresar ilimitadamente la intuición. El tiempo sería, pues, objeto de una intuición que se sabe capaz de extenderse ilimitadamente hacia atrás y hacia adelante, prosiguiendo constantemente una síntesis por definición inacable. Sin embargo esta solución deja pendiente un problema que, a mi modo de ver, no se resuelve sin suponer una cierta dimensión de realidad del tiempo. El problema es éste: ¿cómo puede denominarse intuición de un objeto a la conciencia de una posibilidad? $\mathrm{O}$, mejor aún, a la conciencia de una imposibilidad, porque no podemos suponer que nuestra intuición no pueda extenderse ilimitadamente. Si tenemos la conciencia de tener que extender ilimitadamente nuestra intuición del tiempo, ello se debe a que presuponemos que el tiempo mismo es una magnitud ilimitada. Ahora bien, esta presuposición no resulta de un conocimiento directo del tiempo, sino de nuestra experiencia de la perpetua transformación de los objetos.

En conclusión, el análisis crítico de las exposiciones metafísica y trascendental abre a la interpretación reflexiva del pensamiento kantiano la posibilidad de pensar como convergentes el carácter de intuición formal a priori del tiempo y un tiempo real (el tiempo cósmico) no reductible a una intuición del sujeto finito, pero que hace no sólo posible, sino necesaria esta intuición de acuerdo con sus peculiares características de infinitud, linealidad y unidimensionalidad $u$ homogeneidad. Ciertamente Kant no afirma esta realidad del tiempo, muy por el contrario, explícitamente niega que el tiempo "exista por sí o convenga a las cosas cómo determinación objetiva"13, ni tampoco se refiere a una vivencia del tiempo en sí. Sin embargo, es posible pensar que este tiempo cósmico está presupuesto, pero lo está como forma de la intuición y no como realidad nouménica. $\mathrm{Y}$, a mi modo de ver, es necesario pensarlo así para explicar el hecho de que la sensibilidad necesariamente sea temporal y de que la intuición del tiempo tenga a priori necesariamente la forma que ella tiene. Ahora bien, en tanto pre-supuesto el tiempo es a priori

12 Cf. R. Torreti, op. cit., pp 184-185. Además Torreti cita una serie de textos extraídos de los papeles póstumos de Kant que corroboran su interpretación. ${ }^{13} \mathrm{CRP}$, p. $48, \mathrm{Kr} V$, B 48. 
respecto de los objetos de la experiencia, pero en tanto no tenemos una vivencia de él (para usar la expresión de Ricoeur, en tanto resulta invisible), sólo es captable en función de los movimientos o transformaciones de los objetos, es decir, sólo está dado en nuestra efectiva intuición de objetos. Y si hacemos abstracción de nuestra intuición subjetiva, el tiempo en sí mismo no es nada ${ }^{14}$. Por ello dijimos que este tiempo real o cósmico está presupuesto como forma de la intuición y no como realidad nouménica. Por ello también soy de la misma opinión que Ricoeur, cuando afirma que el acento principal de la exposición metafísica "está puesto en el carácter de presuposición de toda aseveración sobre tiempo", pero inmediatamente agrega que "este carácter es inseparable del estatuto relacional y puramente formal del tiempo"15. Realidad presupuesta e idealidad intuitiva del tiempo son así convergentes y no contradictorias.

Ahora bien, hemos afirmado en la introducción que en Kant no sólo conviven la idealidad y la realidad del tiempo, sino que este tiempo invisible, presupuesto como forma a priori de la intuición de todo fenómeno en general, esconde u oculta una fenomenología en ciernes del tiempo como tiempo de la conciencia. Dicho de otro modo, el tiempo, que en principio parecería ser tan sólo una forma a priori de la intuición de los objetos de la experiencia y que sería caracterizable por su homogeneidad de instantes cualesquiera y por su linealidad unidimensional, oculta, como punto de partida de la constitución de la propia intuición temporal objetiva, una autorreferencia subjetiva de la conciencia a su propio decurso y, por tanto, un ahora o presente diferenciado con sus respectivos horizontes de pasado y futuro. Para decirlo concretamente (aun cuando esto pueda parecer una provocación para las interpretaciones más tradicionales): la intuición del tiempo como forma a priori de la experiencia de objetos es ya una temporalización de un tiempo cósmico que hay que presuponer, aunque en sí mismo sea inexperimentable, y esa temporalización tiene como punto de partida oculto una experiencia del decurso temporal no como forma de percibir los objetos empíricos, sino como forma del propio decurso de la conciencia. El tiempo no sería sólo intuido a través de los objetos de la experiencia, sino, primeramente en la conciencia del fluir de la propia conciencia. Y como esa conciencia es "ahora", la temporalización del tiempo

\footnotetext{
${ }^{14}$ Cf. $K r V$, A 35.

${ }^{15}$ TN III, p. 698.
} 
como una secuencia de instantes cualesquiera unidimensionales implicaría un presente y sus respectivos horizontes temporales. ¿Hasta qué punto la estética nos ofrece un pie para esta suposición?

En primer lugar, como señala Ricoeur, es necesario presuponer la idea del tiempo como una proyección extática de un horizonte temporal tanto de futuro como de pasado para poder "compensar el carácter fragmentario de toda experiencia del tiempo"16. En efecto, la representación de la sensibilidad de un tiempo homogéneo como forma que acompaña la intuición de todos los objetos implica la idea de la continuidad del tiempo. Pero nuestra intuición de objetos jamás puede darnos la idea de un tiempo continuo, porque se trata siempre de una experiencia fragmentada, que se desarrolla paso a paso y que supone y no fundamenta la continuidad del tiempo. Así, el progreso científico, es decir, el progreso en la intuición objetiva, no es lo que nos brinda la continuidad del tiempo, justamente porque el progreso es discontinuo, supone rupturas, avance fragmento por fragmento, sin tener nunca su objeto integralmente ante la mirada determinativa, sino que él supone esa continuidad. Esta continuidad supuesta por nuestra intuición temporal de los objetos no puede consistir, pues, sino en la proyección de un horizonte temporal que, en tanto horizonte, sólo puede ser constituido a partir de un ahora extraible no de los momentos cualesquiera del tiempo como forma pura de la intuición, sino de la autorreferencia de la conciencia a su propio presente. Esta idea de horizonte, que implica la tridimensionalidad del tiempo, subyace oculta tanto al primer argumento de la estética, que quiere que la idea del tiempo esté supuesta por cualquier experiencia de las relaciones de los objetos en el tiempo, cuanto, como lo adelantamos más arriba, al segundo argumento, que cree posible la experiencia de un tiempo vaciado de todo contenido objetivo. Incluso en el caso del tercer argumento que afirma que el tiempo es un singular único y que sólo se pueden distinguir partes y no especies suyas pareciera estar guiada por la idea de horizonte ${ }^{17}$. Pero es el cuarto argumento, el que considera al tiempo como una magnitud infinita dada, el que más aboga a favor de la tesis de Ricoeur, que personalmente comparto, de una fenomenología oculta en la idea del tiempo como una forma pura unidimensional y trascendental de la intuición. En efecto, ¿cómo es posible limitar partes de un tiempo infinito e indefinido, si

${ }^{16}$ TN III, p. 701.

${ }^{17}$ Cf. ibid, especialmente nota 45. 
no es a partir del ahora en que somos conscientes de nuestra intuición y, de ese modo, introducimos una diferenciación y dimensiones en la sucesión de los instantes iguales? Así como el tercer argumento, que nos hablaba de un tiempo único y total que engloba todas sus partes (y que nunca esta dado en la intuición de objetos) supone la idea fenomenológica de horizonte, así también el cuarto que nos habla de las partes del tiempo como limitaciones de una magnitud infinita dada supone el ahora fenomenológico a partir del cual se establecen los límites. En efecto, aun cuando renunciemos a equiparar el desde donde de la representación limitativa al presente viviente husserliano, "no podemos sino interrogarnos sobre el estatuto de la representación por medio de la cual esta limitación es captada"18. Si tenemos en cuenta la dimensión fenomenológica oculta en los argumentos de la estética, tenemos que coincidir con Ricoeur cuando afirma que la demostración de la idealidad del tiempo supone una fenomenología implícita en la experiencia del fenómeno ${ }^{19}$. Pero tenemos que ser más radicales que el propio Ricoeur y coincidir con Heidegger cuando afirma que "el tiempo, como afección pura de sí mismo, no se presenta <junto> a la apercepción pura <en el espíritu>"20, sino que "el si-mismo finito y puro tiene en sí un carácter temporal"'21.

Pero si en esto concuerdo con Heidegger, disiento en un todo con él cuando, negando una de las dos aristas de la primera aporía de la temporalidad y reduciendo el tiempo cósmico a la temporalización del tiempo por el sí mismo, afirma: "el yo no puede ser concebido como temporal, es decir, como intratemporacial, precisamente porque el sí mismo es originariamente, conforme a su esencia íntima, el tiempo mismo"22. Afirmar que la concepción del tiempo como forma a priori de la intuición implica una fenomenología del tiempo como autoexperiencia de la conciencia de su propio decurso no significa suprimir el estrato profundo del tiempo cósmico y sostener a secas que "el sí mismo es el tiempo mismo". Es cierto que la intuición de los objetos en el tiempo supone como punto de partida de la

\footnotetext{
18 Ibid.

${ }^{19}$ Cf. TN III, p. 702.

20 Martin Heidegger, Kant y el problema de la metafísica, trad. Gred Ibscher Roth, México, FCE, 1954, p. 161.

21 Ibid:

22 Martin Heidegger, op. cit., p. 164.
} 
constitución de un horizonte temporal continuo el "ahora que"en el cual los fenómenos son intuidos por un existente que trata con ellos, y, en tanto tal, supone el tiempo de la conciencia o tiempo fenomenológico, es decir, un ahora diferente de los momentos cualesquiera, dado por el instante de la autoconciencia en la intuición del objeto. Pero no es por ello menos cierto que el becho de que de modo necesario tengamos que intuir los objetos temporalmente y constituir un horizonte temporal ilimitado y lineal a partir del ahora de la autocociencia se debe a la constante transformación efectiva del universo y presupone un tiempo cósmico o real. Pero lo presupone como forma de la intuición y no como objeto. Dicho en términos inevitablemente técnicos: toda temporalización del tiempo, incluso la kantiana, para la cual el tiempo equivale a una sucesión homogénea de instantes cualesquiera como forma a priori de la intuición del objeto, implica la autoexperiencia temporal del sí mismo, y, por ende, las dimensiones diferenciadas de presente, pasado y futuro; pero la temporalización no es el "tiempo mismo", sino el modo en que intuimos o recibimos un hecho que se nos impone, a saber, el proceso de transformación del universo, cuya manifestación por excelencia es el movimiento astral. Este proceso efectivo de transformación constituye la condición última de la intuición temporal de los objetos y da testimonio del tiempo como distinto de la temporalización, como residuo invisible del proceso de transformación y no eliminable a través de idealización alguna, pues ninguna idealización puede ni poner en movimiento ni detener el proceso, a lo sumo con-figura el modo en que lo recibimos. Este tiempo efectivo (correlato noemático de la temporalización) que la intuición no produce, sino que padece y que se ve limitada a experimentar, no es, repetimos, asible en sí mismo, pues, inevitablemente, en cuanto tomamos conciencia de él lo constituimos en un ahora de la conciencia, en tiempo temporalizado y, por tanto, lo remitimos a las dimensiones de presente, pasado y futuro, que son tales respecto de una conciencia. Pero este tiempo está ahí, le pasa a la propia intuición del espíritu finito, al punto que es finito, que muere y que deja de intuir; y pasa independientemente de toda intuición de un espíritu finito, a menos que asumiendo un idealismo principista neguemos el proceso de transformación universal del que nos hablan las ciencias y que precede a la emergencia del hombre en el cosmos; a menos que neguemos que vamos envejeciendo, que, con independencia de nuestra intuición, nuestras células se oxidan. Sobre esta irreductibilidad del tiempo a un producto subjetivo, a la temporalización, da testimonio, por sobre todo, la concepción del tiempo en la analítica trascendental, particularmente en las 
analogías de la experiencia. En ella conviven por antonomasia sintetizadas las dos aristas de la primera aporía.

\section{Las analogías de la experiencia}

\subsection{La realidad ideal del tiempo}

"La doctrina del esquematismo trascendental explica las condiciones generales bajo las cuales únicamente podemos emplear conceptos puros del entendimiento en juicios sintéticos"23. Ella da origen a los principios del entendimiento puro: aquellos principios por medio de los cuales es posible subsumir toda percepción bajo los conceptos puros del entendimiento (categorías). Se trata, pues, de principios que constituyen "reglas del uso objetivo de las categorías"24. Y la esquematización es precisamente lo que nos permite utilizar objetivamente una categoría. Pero, ¿qué habremos de entender por esquematización de un concepto puro? La respuesta es breve: su sensibilización. Ahora bien, si tenemos en cuenta que "se llama sensibilización a la manera mediante la cual un ser finito puede hacer algo intuible para sí mismo"25, entonces cabría preguntarse de qué manera se vuelven intuibles los conceptos puros del entendimiento para poder subsumir en ellos toda percepción. Pues bien, los conceptos puros del entendimiento en tanto puros necesitan ser intuibles de un modo esencialmente puro y su aspecto sensible (su imagen) no puede extraerse nunca del ámbito de lo intuible empíricamente. Kant lo afirma expresamente: "El esquema de un concepto puro del entendimiento es algo que no puede ser puesto en imagen alguna"26. Las categorías, en tanto conceptos trascendentales, no pueden ponerse en imágenes empíricas, porque ellas justamente representan las condiciones dentro de las cuales es posible encontrarse con un objeto empírico en tanto que tal. Por lo tanto, si los conceptos puros han de ser intuibles para que en ellos se puedan subsumir las percepciones objetivas, y si esa intuición no puede ser empírica, el esquema de un concepto puro ha de resultar de la síntesis (operada por la imaginación trascendental) entre tales conceptos y las intuiciones puras,

\footnotetext{
${ }^{23}$ R. Torreti, op. cit. p. 428.

${ }^{24} \mathrm{CRP}$, p. 106, KrV A 161/ B 200.

${ }^{25}$ M. Heidegger, op. cit. p. 82.

${ }^{26}$ CRP, p. 99; KrV A 142/ B 181.
} 
particularmente el tiempo, en tanto éste constituye la imagen pura, es decir, la forma en que, con independencia de toda experiencia, nos imaginamos todos los objetos de los sentidos en general. "El tiempo, pues, en calidad de intuición pura es algo que proporciona un aspecto antes de toda experiencia"27. Por ello se puede llamar a la temporalización de las categorías, esto es, a la introducción de las distintas perspectivas o respectos desde los cuales es posible representarse un objeto en la sucesión de momentos consecutivos, imagen pura o esquema de los conceptos puros del entendimiento. Consecuentemente es posible afirmar que "el tiempo, como 'imagen pura', es la imagen-esquema, y no solamente la forma de la intuición opuesta a los conceptos puros del entendimiento"28.

Ahora bien, en tanto esquematizado, el tiempo resulta determinado. Los esquemas son principios trascendentales que regulan la constitución de toda experiencia de objetos. Y como el tiempo es sólo una forma a priori de la intuición de objetos, de ello se deduce que los esquemas regulan o determinan el tiempo (la sucesión infinita de momentos cualesquiera) determinando las reglas de toda experiencia de los objetos en el tiempo. Por ello puede afirmar Kant que "los esquemas no son nada más que determinaciones a priori del tiempo, según reglas (...)"29. Dado que estos esquemas temporalizan (sensibilizan) los conceptos puros del entendimiento, haciendo surgir así los principios del entendimiento puro, y dado que los conceptos puros son extraidos por Kant de la tabla de los juicios, resulta que los principios del entendimiento puro mostrarán cuatro tipos generales de determinación del tiempo concordantes con los cuatro grandes tipos de juicios (cantidad, cualidad, relación y modalidad) y, consecuentemente, con los cuatro momentos de la división de las categorías. Esos cuatro modos fundamentales de determinación del tiempo son los siguientes: "serie del tiempo, contenido del tiempo, ordenación del tiempo y conjunto del tiempo con respecto a todos los objetos posibles"30. Estas determinaciones enriquecen la noción proveniente de la estética del tiempo como sucesión infinita de momentos homogéneos, pero no implican, por lo menos expresamente, una fenomenología del tiempo que incluya un presente vivido

\footnotetext{
${ }^{27}$ M. Heidegger, $o p$. cit., p. 92.

${ }^{28}$ Ibid.

${ }^{29} \mathrm{CRP}$, p. 100; KrV A 145/ B 184.

${ }^{30} \mathrm{Ibid}$.
} 
y sus relaciones con el pasado y con el futuro mediante la protensión y la retención, sino que se trata de una determinación indirecta de las características universales del tiempo a partir de las leyes a priori que regulan nuestra experiencia de las relaciones en el tiempo entre los fenómenos.

De los cuatro tipos de principios del entendimiento puro son las analogías de la experiencia, es decir, aquellos principios que se refieren a la determinación del tiempo según su ordenación, los que Kant ha desarrollado más detenidamente y los que resultan más significativos para la comprensión de la convergencia de idealidad y realidad del tiempo. Las analogías de la experiencia incluyen los esquemas de sustancia, causalidad y comunidad o acción recíproca entre los fenómenos, gracias a los cuales se puede hacer surgir de la multiplicidad de percepciones fenoménicas la unidad de la experiencia. El principio general de las analogías es, según su formulación en la primera edición de la Crítica, el siguiente: “Todos los fenómenos están sujetos a priori, según su existencia, bajo reglas de la determinación de sus relaciones mutuas en un tiempo" 31 . Para la correcta comprensión de este principio hay que tener en cuenta, en primer lugar, que las relaciones en el tiempo de todos los fenómenos que constituyen la experiencia científica son representadas objetivamente, esto es, son las mismas para todo sujeto empírico; y, en segúndo lugar, que "el tiempo mismo no puede ser percibido"32. De los dos puntos precedentes se deriva que la determinación del carácter objetivo de las relaciones temporales de los objetos de la experiencia no puede provenir de una experiencia directa del tiempo, y como, obviamente, tampoco puede provenir de lo múltiple de la intuiciones particulares, que se refieren unas a otras de modo meramente casual, habrá que concluir que tal carácter objetivo de las relaciones de los fenómenos en lo que respecta a su existencia en un tiempo dado se deriva de principios (las analogías) que enlazan a priori y, por tanto, de modo universal y necesario, la conjunción de lo múltiple de la intuición empírica con en una única $y$ determinada ordenación del tiempo. Dicho de otro modo: las características o determinaciones universales del tiempo, a la que están sujetos necesariamente todos los fenómenos, tal cual lo exige la unidad trascendental de la

${ }^{31} \mathrm{KrV}$ A 176 (trad. mía). En la segunda edición el principio es enunciado en estos términos: "La experiencia sólo es posible a través de la representación de un enlace necesario de las percepciones" KrV B 218 (trad. mía).

${ }^{32}$ CRP , p. 113; KrV B 219. 
apercepción, no resultan de la percepción directa o pura del tiempo, sino de leyes a priori de las relaciones entre los fenomenos en el tiempo. Dichas leyes son las analogías que pueden ser caracterizadas, entonces, como "reglas de la determinación general del tiempo"33.

La más importante de las observaciones sobre el tiempo nos la brinda la primera analogía: "el principio de la permanencia de la sustancia". Para comprender el sentido de esta primera analogía, como también el de las dos siguientes, hay que partir de la observación previa de Kant según la cual tres son los modos del tiempo: permanencia, sucesión y simultaneidad que se corresponden con las tres reglas a priori que determinan las relaciones de tiempo entre los fenómenos empíricos "con respecto a la unidad de todo tiempo" 34 . La expresión "con respecto a la unidad de todo tiempo" indica que las tres reglas del caso (las analogías), determinando a priori las relaciones de tiempo entre los fenómenos, determinan a su vez los modos objetivos (universales y necesarios) de darse del tiempo para todo sujeto. Así "el principio de permanencia de la sustancia" determina como un modo necesario del tiempo su permanencia, es decir, determina el tiempo como un solo tiempo continuo que, por decir así, siempre "esta ahí", pues si la sustancia permanece debe haber un tiempo en el cual su quantum permanezca siendo el mismo a lo largo de todos los cambios. Este principio en la segunda edición de la Crítica es formulado en estos términos: "En todo cambio de los fenómenos permanece la sustancia y el quantum de la misma no aumenta ni disminuye en la naturaleza"35. La prueba podría resumirse del siguiente modo: como todos los fenómenos están en el tiempo han de ser sucesivos o simultáneos. Ahora bien, estas relaciones sólo pueden establecerse respecto de un único tiempo que permanece y no cambia lo largo del cambio de los fenómenos. Pero resulta que el tiempo por sí mismo no puede ser percibido $\mathrm{y}$, por tanto, no puede ser tomado como término de referencia para las relaciones de sucesión y de simultaneidad. Por tanto, para que tales relaciones puedan establecerse y para que pueda llegar a conocerse que efectivamente el tiempo permanece, es necesario encontrar en los fenómenos mismos ese término de referencia. Es necesario, pues, hallar en los propios fenómenos algo idéntico a sí mismo y permanente, que representa la

\footnotetext{
${ }^{33} \mathrm{CRP}$, p. 114; $\mathrm{KrV}$ A 178/ B 220.

${ }^{34} \mathrm{CRP}$, p. 114; $\mathrm{Kr} V$ A $177 / \mathrm{B} 219$.

${ }^{35}$ CRP, p. 115; KrV B 224.
} 
permanencia y unicidad del tiempo. "Debe hallarse el sustrato que representa el tiempo en general" 36 . Este sustrato es, para Kant, la permanencia de un quantum de sustancia en el todo de la naturaleza. Ciertamente Kant, a través de la prueba de la primera analogía, demuestra la necesidad de distinguir en los fenómenos un aspecto cambiante y uno permanente y referir aquél a éste, empero no parece tan claro que se haya demostrado que lo permanente sea el quantum de la sustancia, aun cuando se interprete este quantum como una magnitud invariable de masa. En efecto, la física contemporánea ha demostrado que la masa es una función de la velocidad y un estado de la energía, y pudo formular una ecuación entre masa y energía, por lo que es la energía la que vino a concebirse como aquella magnitud cuya distribución no es homogénea, pero cuya suma se mantiene invariable en un sistema cerrado. Este último punto representa una grave dificultad para la tesis kantiana, puesto que la primera analogía sólo valdría en una región cerrada del universo. Ahora bien, en el tiempo unitario y permanente, al igual que en el universo, no puede haber tales regiones cerradas, por lo que la primera analogía sólo puede valer para el todo del universo y no para sus partes. Sin embargo, como la primera antinomia de la razón pura lo declara, es imposible determinar si el universo es un sistema cerrado o abierto. De todo ello resulta que el principio de conservación de la sustancia no puede tener un valor constitutivo, sino, como el propio Kant lo declara, uno regulativo ${ }^{37}$, en cuanto amplía indefinidamente por medio del progreso de la experiencia nuestro conocimiento del universo abordado como un sistema que se supone cerrado pero que no resulta nunca asible en su totalidad. Sin embargo, aunque este principio es sumamente útil para el progreso del conocimiento del universo, parece no brindarnos el elemento o sustrato que necesitábamos para justificar la permanecia del tiempo, la cual, por otra parte, es imprescindible suponer para ordenar con respecto a él la sucesión de fenómenos. Por lo tanto, la pregunta se impone: ¿de dónde viene este modo necesario del tiempo que es su permanencia? ¿Percibimos el tiempo como permanente por la supuesta permanencia de la sustancia o, por el contrario, porque el tiempo real se nos impone como un único tiempo continuo percibimos tanto los cambios como sucesivos cuanto la relativa invariabilidad de ciertas magnitudes? ¿La afirmación de que la permanencia

\footnotetext{
${ }^{36} \mathrm{CRP}$, p. 116.

${ }^{37}$ Cf. CRP, p. 115; KrV A 179/ B 222.
} 
del tiempo la conocemos a través de la permanencia de la sustancia (lo que, por su parte, como vimos, no es ningún conocimiento) no es sino un rodeo para afirmar que necesitamos suponer un tiempo único, que no pasa y en el que todo pasa, para dar cuenta de la efectiva sucesión de los cambios y la permanencia de la energía en una región arbitrariamente cerrada del universo? ¿Y no resulta esta necesidad del modo en que el tiempo mismo hace que se den los fenómenos? Tocamos aquí un punto de gran profundidad. Es cierto que no percibimos la permanencia por sí misma, sino una cantidad de tiempo en la cual dura un sustrato (por lo cual no tenemos acceso directo al tiempo, sino mediado por los objetos en el tiempo), pero no es menos cierto que no podemos no experimentar que un sustrato dura, porque ya siempre suponemos la permanencia del tiempo. Según mi modo de ver la cuestión, no se puede resolver el problema que plantea la primera analogía - la necesidad de un tiempo permanente como sustrato del cambioen función de suponer otro sustrato cuya cantidad no cambia. En primer lugar, porque tal sustrato, como la muestra la física contemporánea, es imposible de determinar. Y, en segundo lugar, porque no se deduce del principio a priori de la permanencia de la sustancia la permanencia del tiempo. $\mathrm{Si}$ así fuera, sería una permanencia meramente ideal. Por el contrario, en la afirmación de la permanencia de la sustancia, se esconde la certeza a priori de que el tiempo considerado como sustrato constante de los cambios realmente permanece como tiempo de la naturaleza independientemente mío o de otro espíritu finito. Personalmente me parece que esta dimensión de realidad del tiempo, a la que nos conduce el estudio crítico de la primera analogía, está latente en los análisis del propio Kant. En efecto, Kant señala que la permanencia del sustrato "representa (vor-stellt) el tiempo en general", es decir, la permanencia del tiempo único, que, por tanto, parece estar supuesta como real, y no que dicha permanencia constituya su condición de posiblidad. Además, al comienzo de su análisis de las analogías Kant afirma que "los tres modos del tiempo son: permanencia, sucesión y simultaneidad. De alli que (Daber) haya tres reglas de todas las relaciones de tiempo de los fenómenos" 38 , es decir, en el propio lenguaje de Kant se oculta el hecho de que no son las reglas las que determinan idealmente el tiempo, sino que es el tiempo en su realidad inasible el que determina y posibilita las las reglas. Esta dimensión real presupuesta del tiempo en Kant debe ser considerada

${ }^{38} \mathrm{KrV}$ A 176/B 219 (traducción y cursivas mías). 
seriamente. Es porque el tiempo realmente permanece que podemos suponer que en un sistema cerrado a lo largo del tiempo se mantiene constante la sustancia, ya sea que se la interprete como quantum de masa o de energía, y no es que el tiempo permanezca porque haya un espíritu finito que piensa la sustancia como invariable. Si lo último fuera cierto habría que colegir que sólo en cuanto pensada por un individuo la sustancia permanece y que antes o después de la existencia de un espíritu finito ella podría perfectamente escaparse hacia la nada o surgir desde esa misma nada. Sin embargo, también es cierto que hay una dimensión ideal del tiempo en cuanto esta permanencia sólo es captada a través de el principio ideal de la permanencia de la sustancia. Pero una cosa es que en la permanencia de la sustancia (o, al menos, en la duración de un sustrato) advirtamos la permanencia del tiempo, y muy otra suponer que es la idea de la permanencia de la sustancia la que produce la permanencia del tiempo. Hay, pues, en la primera analogía una peculiar convergencia entre realidad e idealidad del tiempo en cuanto hay una estricta correspondencia "entre la determinación del tiempo (la inmutabilidad) y la determinación de las apariencias según el esquema (la permanencia de lo real en el tiempo"39. Precisamente en el esquema es donde convergen la dimensión de realidad - la permanencia del tiempo- y la dimensión de idealidad- la representación de lo empírico como sustrato de la determinación del tiempo en general.

La segunda analogía es denominada "principio de la sucesión según la ley de la causalidad" 40 . Ella representa ese otro modo necesario del tiempo que es, precisamente, la sucesión; o, para ser más exactos, ella confiere al orden del tiempo la determinación de sucesión regular. En la segunda edición de 1787 se la enuncia en estos términos: "Todas las transformaciones suceden según la ley del enlace entre causa y efecto" 41 . En el enunciado de la edición de 1770 Kant expone cómo comprende el principio de causalidad: "Todo lo que ocurre (comienza a ser) supone algo anterior a lo cual sigue según una regla"42. La prueba del principio podría resumirse de este modo: como el tiempo mismo no puede ser percibido, la sucesión no puede establecerse directamente por comparación con él, sino por un enlace causal tal entre los

\footnotetext{
${ }^{39}$ TN III, p. 705.

${ }^{40}$ CRP, p. 119; KrV B 233.

${ }^{41}$ Ibid.

${ }^{42} C R P$, p. 119; KrV A 189.
} 
fenómenos que el surgimiento de uno supone necesariamente otro que le precede. Así como en la primera analogía la permanencia del tiempo sólo podía ser pensada sobre la base de la permanencia de la substancia, en la segunda la sucesión sólo puede ser representada por la síntesis causal a priori operada por el yo pienso. Kant hace la salvedad de que "la mera sensación deja indeterminada la relación objetiva de los fenómenos sucesivos"43, puesto que la imaginación puede enlazar arbitrariamente dos fenómenos y hacer preceder tanto a uno como al otro en el tiempo. Ello es así precisamente porque el tiempo no puede ser percibido por sí mismo y, por tanto, en relación con el tiempo puro no puede determinarse cuál fenómeno es primero y cuál después. Para que la relación de sucesión sea objetiva no basta pues que aleatoriamente, mi imaginación ponga una sensación antes y otra después, sino que es necesario que un objeto preceda al otro. Y ello es sólo posible si los dos objetos son enlazados de un modo tal que, en virtud del enlace, quede determinado con absoluta necesidad cuál de ellos debe ponerse antes y cuál después. El principio que opera dicha unidad sintética es el de causalidad, en cuanto la causa determina en el tiempo el efecto como subsecuente y no como algo que meramente pudiera ser puesto después en el orden de la imagen. Se trata en el principio causal de una sucesión en la que conozco que B "realmente sucede" 44 a A, porque B es el efecto de la causa A. Excedería los límites de nuestro análisis discutir en detalle este principio. Baste con decir que la nueva mecánica y el llamado principio de indeterminabilidad, que advierte que no es posible asignar simultáneamente una posición y una trayectoria (momento cinético) determinados a una particula de materia, han puesto en cuestión la pretensión, implícita en el principio de causalidad, de que, conocido un fenómeno, podría determinarse aquel que le sucede. De allí que la nueva mecánica, hable de la probabilidad de que, en los sistemas que estudia, dado un hecho le suceda otro, pero no determina la sucesión de hechos singulares efectivamente observables. La idea de que todo estado de cosas es causa de un cierto efecto sería, pues, también un principio regulativo que sirve para ampliar nuestro conocimiento de las relaciones entre los fenómenos y aumentar constantemente el grado de probabilidad de nuestras predicciones. Pero se trata de un supuesto: suponemos que a un estado de cosas A le sigue el estado de cosas B como su 
efecto, pero no es posible determinar por anticipado con absoluta necesidad cómo será $\mathrm{B}$ y, por tanto, no es posible decir que $\mathrm{B}$ es el necesario efecto de A. Se genera, pues, en torno del principio de causalidad una situación análoga al de permanencia de la sustancia: no es posible afirmar que suponemos que el tiempo es un continuo porque dados dos fenómenos estos necesariamente se siguen de acuerdo con una relación causal, puesto que toda sucesión causal es sólo probable, mientras que el carácter continuo del tiempo es necesario: un estado de cosas se sucede continuamente a otro aunque no podamos determinar una relación causal efectiva entre ambos. Mas bien pareciera que, porque realmente el tiempo es continuo, podemos suponer que es posible enlazar los fenómenos de acuerdo con un vínculo causal. Pero, por otra parte, también es cierto que ese carácter continuo lo advertimos no por una experiencia directa de la continuidad del tiempo en sí, sino que lo leemos precisamente en el carácter causal de una naturaleza que responde a la idea que Newton se ha forjado de ella ${ }^{45}$.

No es, entonces, la causalidad la que genera o produce el carácter continuo del tiempo, sino que ella lo presupone como su condición de posibilidad, aun cuando se trate de una condición inasible en sí misma. Pero precisamente porque es inasible en sí es que a través del principio ideal de la sucesión causal de los fenómenos advertimos el carácter continuo del tiempo. Realidad e idealidad convergen así en el principio de causalidad ${ }^{46}$. Si

45 TN III, p. 706.

${ }^{46}$ Aun cuando luego su interpretación avanza por carriles diferentes, también Joseph Moreau se ve obligado a distinguir entre un tiempo real, el tempus aequaliter fluit de Newton, que hace posible la causalidad, y el tiempo de la representación objetiva causal, aunque deja en claro que lo que fluye no es el tiempo, cuya continuidad es, por así decir, inmutable, sino los objetos en él. Escribe Moreau "Este tiempo inmutable, es aquel que interviene en las ecuaciones de la mecánica, por el cual se bace posible la representación objetiva, la determinación matemática del movimiento; él es representado por una línea, y a los puntos que se pueden distinguir en esta línea corresponden instantes sucesivos en el tiempo." Joseph Moreau, "Le temps de la représentation", en: Les etudes pbilosopbiques (3), 1980, pp 273-284, aquí p. 274 (cursivas mías). Otro intérprete de Kant, Friedrich Kümmel ha notado esta convergencia de realidad e idealidad del tiempo, sólo que en lugar de interpretarla como convergencia de dos dimensiones que se necesitan mutuamente - el tiempo real al que estamos sometidos y la temporalización del tiempo como modo libre de espontaneidad cognoscitiva - la ha interpretado como ambigüedad en el concepto de 
quisiéramos eliminar este residuo invisible de realidad necesario para poder presuponer el principio de causalidad, debiéramos concluir que es la idea de causalidad, es decir, el hecho de que un espíritu finito enlace causalmente dos fenómenos, no lo que permite advertir la continuidad del tiempo real, sino lo que la produce. Consiguientemente habría que pensar que no habiendo tal espíritu que vincule causalmente, por ejemplo, el surgimiento de los Andes con la aparición de los grandes lagos patagónicos, ello significaría que el tiempo del primer hecho no se continúa con el del segundo. Es decir, podría pensarse que los dos hechos ocurrieron "juntos" o que entre los dos no habría continuidad alguna del paso del tiempo, precisamente la continuidad necesaria para que se llenaran los lagos. Tal imaginería va en contra del propio Kant, quien dice explícitamente respecto de lo que se sucede que "sucede realmente (wirklich)" 47 .

La tercera analogía, que la segunda edición de la Crítica denomina "principio de la simultaneidad según la ley de la acción recíproca o comunidad" 48 , se enuncia en esa misma edición en estos términos: "Todas las substancias, en cuanto pueden ser percibidas en el espacio como simultáneas, están en universal acción recíproca"49. Ella da cuenta del tercer modo necesario del tiempo: la simultaneidad. Su prueba es análoga a las dos anteriores: como no puedo percibir el tiempo en sí mismo, sólo puedo decir que dos cosas o sustancias son simultáneas en cuanto interactúan, "pues sólo bajo esa condición pueden las referidas sustancias ser representadas empíricamente como simultáneamente existentes" 50 . No es, pues, la

tiempo. Escribe Kümmel: "Como condición del conocimiento ellos [tiempo y espacio] se vuelven para Kant formas de la manifestación de la libertad misma, que se empuña y se afirma a sí misma en su síntesis [idealidad del tiempo]. Pero al mismo tiempo se afirma una noción de tiempo como plexo general legal causal que excluye de sí la libertad [realidad del tiempo]. En esta inconciliable contradicción de una libertad que constituye el conocimiento como sistema necesario y que a la par se excluye de este [sistema] se expresa con claridad la ambivalencia del concepto de tiempo subyacente." Friedrich Kümmel, "Zeitbewußtsein als Einheit von Spontaneität und Rezeptivität", en: Zeitschrift für philosophische Forschung, Januar-März 1972, Band 26-Heft 1, pp. 21-28, aquí p. 28.

${ }^{47}$ Cf. $\mathrm{KrV}$ A 202/ B246.

${ }^{48} \mathrm{CRP}$, p. $128 ; \mathrm{KrV}$ B 256

${ }^{49} \mathrm{Ibid}$.

${ }^{50} \mathrm{CRP}$, p. 129; $K r V$ A 212/ B 259. 
sensación simultánea de dos fenómenos lo que garantiza su simultaneidad objetiva, sino un enlace o síntesis entre esos fenómenos regido por un principio a priori que dice que, si dos estados de dos substancias se determinan mutuamente en virtud de la interacción recíproca de esas substancias, tales estados son simultáneos. También en este caso la física contemporánea ha puesto en cuestión la vigencia universal del principio de interacción. En efecto, tal principio exige, para establecer la simultaneidad de dos fenómenos, una acción causal recíproca instantánea. Pero para la física contemporánea es un hecho que no existen acciones causales instantáneas, pues ninguna acción se propaga a una velocidad mayor que la de la luz en el vacío. En conclusión, el principio de interacción instantánea no es una condición de posibilidad del saber empírico (de hecho la ciencia contemporánea prescinde de él), y no es posible la simultaneidad absoluta entre fenómenos no contiguos. La simultaneidad es sólo relativa al marco de referencia que se elija. La comunidad de fenómenos en el espacio sólo puede construirse retrospectiva o prospectivamente cuando las consecuencias de ambos hayan afectado o vayan a afectar en un mismo momento a un mismo marco referencial, pero no tiene sentido hablar de una simultaneidad de los objetos con respecto a un instante dado ${ }^{51}$. De allí que podamos concluir que un instante de tiempo es en sí mismo (realmente) simultáneo con otro instante o, para ser más precisos, con ese mismo instante en tanto inuido por otra conciencia, y no que dos instantes son simultáneos porque un espíritu finito enlaza dos fenómenos de acuerdo con una sólo presunta y empíricamente falaz interacción mutua instantánea. No es la inexistente interacción mutua instantánea entre dos fenómenos lo que produce instantes simultáneos, sino que, por el contrario, porque a una pluralidad de fenómenos les pasa realmente el mismo tiempo (en cuanto los transforma de modo efectivo) o pasan en el mismo tiempo, es que ellos pueden ser relativamente simultáneos respecto de un marco de referencia. Pero si esto es cierto, no lo es menos que "leemos" el "pasar" simultáneo del tiempo no en sí mismo, sino en la relativa simultaneidad de dos fenómenos cuyos efectos resultan concomitantes para un marco de referencia. Realidad e idealidad convergen así también en el principio de interacción mutua.

Así como el análisis crítico de la estética nos llevó a afirmar que no es posible sostener una idealidad absoluta del tiempo, sino que se trata de una

${ }^{51}$ Cf. para el desarrollo de esta idea R. Torreti, op. cit., pp. 458-459. 
idealidad real en cuanto un tiempo real está presupuesto como forma de la intuición, conluímos ahora en nuestro análisis de las analogías que ese residuo temporal real, presupuesto por la propias analogías, sólo es experimentado a través de los modos en que nuestro pensamiento sintetiza idealmente las relaciones temporales entre los fenómenos, por lo que bien podemos hablar de una realidad ideal de la analítica correlativa a la idealidad real de la estética. Esta realidad ideal puede expresarse sintéticamente en dos puntos. El primero mienta la idealidad, el segundo la realidad del tiempo: 1) las relaciones dinámicas de inherencia (substancia), de consecuencia (causalidad) y de composición (acción recíproca), organizando el orden de los fenómenos en el tiempo, organizan a la vez las tres relaciones de orden del tiempo (genitivo subjetivo), que en sí mismo no puede ser percibido, como permanencia, sucesión y simultaneidad ${ }^{52}$; y 2) sin embargo, dichas relaciones no producen el tiempo, sino que son sólo "principios de la determinación de la existencia de los fenómenos en el tiempo, según los tres modos del [genitivo subjetivo] mismo, a saber, la relación con el tiempo mismo como magnitud (la magnitud de la existencia, es decir, la duración), la relación en el tiempo como serie (sucesión) y por último la relación en el tiempo como conjunto de toda existencia (simultaneidad)" 53 .

\subsection{Fenomenología implícita}

¿Se agota la determinación ideal del residuo temporal real en los principios del entendimiento puro? Dicho de otro modo: ¿se reduce la temporalización del tiempo a los principios que regulan las relaciones objetivas entre los fenómenos; o, por el contrario, esta determinación objetiva del tiempo como magnitud de existencia (duración), como serie (sucesión) y como conjunto de toda existencia (simultaneidad) lleva implícita una fenomenología del tiempo y las consecuentes dimensiones de pasado,

52 En términos de Kant: "El tiempo no es considerado como aquello dentro de lo cual la experiencia determine inmediatamente su lugar a cada existencia, cosa imposible porque el tiempo absoluto no es objeto alguno de percepción (...), sino que la regla del entendimiento por medio de la cual tan sólo puede la existencia de los fenómenos recibir unidad sintética, según relaciones de tiempo, determina a cada uno de ellos su lugar en el tiempo, por lo tanto a priori y con validez para todo tiempo." CRP, p 130; KrV A 215; B 262.

53 CRP, p. 130; KrV A 215/ B 262 (cursivas mías). 
presente y futuro, irreductibles a la sucesión infinita de instantes homogéneos?

Como vimos, la analítica trascendental demuestra -y este es para mí el aporte imperecedero de Kant a toda filosofía del tiempo- que el tiempo absoluto no es visible por sí mismo, sino a través del rodeo de los principios del entendimiento puro que regulan el sistema de la naturaleza. De allí que haya una estricta e inevitable correlación entre la determinación del sistema axiomático constitutivo de la ontología de la naturaleza y las determinaciones del tiempo. Ricoeur plantea que "esta reciprocidad entre el proceso de construcción de la objetividad del objeto y el surgir de nuevas determinaciones del tiempo explica que la descripción fenomenológica que podrían suscitar estas determinaciones sea reprimida sistemáticamente por el argumento crítico"54. La hipótesis de Ricoeur parece plausible en cuanto la temporalización del tiempo en las tres analogías implica el propio fluir temporal de la conciencia en tanto consciente de las relaciones temporales de los objetos. Así la permanencia del tiempo, concebida en función de la permanencia de la substancia, es inescindible o indesligable de la proyección de un horizonte de protensiones y de retenciones a partir de la noción de ahora o presente vivo. De lo contrario el tiempo no permanecería, sino que desaparecería y renacería a cada instante. Del mismo modo, en relación con la segunda analogía, podríamos preguntarnos si el criterio que nos permite distinguir entre una sucesión reversible arbitrariamente y otra irreversible está dado únicamente por el principio de causalidad, cuya validez universal se halla seriamente cuestionada por la física moderna, o si, por el contrario, implica la distinción entre un pasado y un futuro a partir del ahora vivo de la autoconciencia. En una teoría como la kantiana para la cual todos los instantes son homogéneos, la distinción entre instante anterior y posterior sólo puede provenir de un criterio exterior al continuo homogéneo que, además, por sí mismo no puede ser percibido. Ese criterio está dado por la representación de la conciencia de la relación de causalidad entre los objetos. Sólo en relación con ese presente de la representación, no reductible a un momento homogéneo (en cuanto es ya la temporalización de un momento tal) pueden dos fenómenos ser anteriores o posteriores, esto es, ser prospectiva o retrospectivamente (jhe aquí de nuevo la proyección del horizonte pro o retencional desde el ahora!) respecto de ese presente futuros

${ }^{54}$ TN III, p. 707. 
o más futuros, pasados o más pasados. Es en relación con este presente vivo de la representación que la intuición de la sucesión o, como dice Ricoeur, "la disimetría entre pasado y futuro" 55 se manifiesta "no constreñible al principio de orden proporcionado sólo por la regularidad causal" 56 . Y todavía de manera más clara la tercera analogía o principio de interacción manifiesta que toda determinación o temporalización del tiempo de acuerdo con principios objetivos de relaciones entre los fenómenos implica una fenomenología del propio fluir temporal de la conciencia, ya que tal principio sólo es posible como contemporaneidad de dos cursos de acción respecto de un marco de referencia dado por el presente viviente en el que un sujeto se representa efectivamente dichos cursos como contemporáneos.

En conclusión, contra Heidegger y Husserl, es necesario afirmar que es imposible hablar de una fenomenología del tiempo puro o de "el tiempo como afección pura de sí mismo" 57 , porque nuestra conciencia interna de existencia implica la conciencia de objetos externos cuyas determinaciones temporales se rigen por principios objetivos que "siguen siendo kantianos" y que, como vimos en el apartado anterior, suponen una realidad empirica del tiempo. Pero, por otro lado, contra Kant, hay que confesar que es necesario suponer una fenomenología implícita sin la cual se rompería el nexo entre las relaciones temporales objetivas y la constitución del tiempo como tiempo efectivo, esto es, como tiempo que le pasa a un sujeto. Sin esta fenomenología no habría manera de distiguir el efectivo paso del tiempo de una mera magnitud matemática abstracta. Por ello es posible afirmar con Ricoeur que fenomenología y crítica tienen una relación complementaria aun cuando tiendan a excluirse mutuamente: "no se puede abarcar con una única y la misma mirada el anverso y el reverso de la misma moneda" 58 .

Ahora bien, que la determinación del tiempo implique una fenomenología de la conciencia del tiempo y que no se agote en los principios del entendimiento puro, en nada obsta para afirmar la realidad "empírica" como supuesta tanto por la concepción del tiempo de la crítica como por la de la fenomenología, porque, como vimos en nuestro análisis de

\footnotetext{
${ }^{55}$ TN III, p. 708.

56 Ibid.

${ }^{57}$ M. Heidegger, op. cit., p. 160.

58 TN III, p 715.
} 
las analogías, esta realidad empírica es la condición de posibilidad que determina la ordenación de los objetos en el tiempo, y porque la conciencia, en tanto ya siempre es consciencia de un sujeto empírico, está sometida a esa misma ordenación en el tiempo real que los otros objetos empíricos. La prueba más clara de ello es la senescencia. Mas la profundización en esta cuestión, a saber, la convergencia entre estos dos modos complementarios de temporalización del tiempo -fenomenología y crítica- y la realidad del tiempo, supuesta por esta temporalización pero inasible si no es a través de ella, exige que demos un último paso en nuestro trabajo y abordemos la refutación kantiana del idealismo.

\section{La refutación del idealismo}

En la segunda edición de la Crítica en 1787 Kant, preocupado por la filiación de su filosofía al idealismo subjetivista de la tradición moderna, intercala en el capítulo dedicado a los postulados del pensamiento empírico un pasaje titulado "Refutación del idealismo"59. La refutación se dirige principalmente contra el idealismo problemático de Descartes que declaró indudable sólo el cogito, es decir, la autoconciencia, y consideró imposible probar por experiencia inmediata cualquier existencia externa diferente de la propia conciencia. La refutación debe, pues, demostrar que de las cosas externas tenemos experiencia efectiva y no mera imaginación, lo cual sólo es posible si se demuestra que la autoconciencia supone la experiencia externa. De acuerdo con ello el teorema kantiano reza: "La mera conciencia, pero empíricamente determinada, de mi propia existencia demuestra los objetos en el espacio fuera de mí"60. La prueba parte de dos premisas. La primera, demostrada en la estética trascendental, afirma que "tengo conciencia de mi existencia como determinada en el tiempo"61, es decir, no tengo autoconciencia a secas sino autoconciencia de estar siendo. La segunda, asegurada por la analítica de los principios, nos dice que toda determinación de tiempo (toda duración) supone algo permanente en la percepción en relación con lo cual dura lo que dura. Esto permanente no puede ser ninguna representación de mi propia conciencia, porque esas representaciones, en

\footnotetext{
${ }^{59}$ Cf. $C R P$, p 134-136; KrV B 274-279.

${ }^{60} \mathrm{CRP}$, p. 135; KrV B 275.

${ }^{61}$ Ibid.
} 
tanto constituyen mi autoconciencia, están sometidas también a una determinación (duración) en el tiempo y "necesitan ellas mismas un substrato permanente distinto de ellas, en relación con el cual pueda ser determinado su cambio y, por consiguiente, mi existencia en el tiempo en el que ellas cambian" 62 . Así pues eso permanente ha de ser una cosa fuera de mí y no una mera representación de una cosa fuera de mí. En otros términos, ha de tratarse de un objeto fenoménico (como, por ejemplo, el movimiento regular de los astros, en función del cual se determina en el tiempo toda duración). "Por consiguiente la determinación de mi existencia en el tiempo es sólo posible por la existencia de cosas reales, que yo percibo fuera de mí. (...). Es decir, que la conciencia de mi propia existencia es al mismo tiempo una conciencia inmediata de la existencia de otras cosas fuera de mí"63. La refutación kantiana retoma el argumento de la primera analogía sólo que lo aplica en un nivel más radical: ya no se trata del plano de la simple representación objetiva de las cosas, sino de la "conciencia de existencia" Naturalmente que esta refutación implica que tales "cosas fuera de mí" puedan existir realmente "como cosas en sî" independientes de mi sensibilidad. Pero ello de ningún modo indica que nosotros las conozcamos en su carácter nouménico ni que las tomemos como referencia de la "determinación en el tiempo" en tal carácter, sino sólo en tanto fenómenos. La refutación del idealismo problemático sólo se limita a mostrar la existencia de cosas externas distintas del sujeto de las representaciones, pero no es una prueba del realismo trascendental: aquellas cosas externas distintas del sujeto que actúan como término de referencia de la determinación en el tiempo de la propia existencia del sujeto le hacen frente a éste como fenómenos, esto es, de acuerdo con la constitución de su propia subjetividad trascendental.

Esta refutación kantiana del idealismo resulta decisiva para nuestra hipótesis de convergencia entre realidad e idealidad del tiempo en Kant en dos sentidos. En primer lugar, muestra que no es posible dar cuenta del fenómeno a través de una fenomenología del tiempo como tiempo puramente conciencial, porque todo acto de autoconciencia remite de suyo, como término de referencia en lo que a la determinación de su duración o trans-curso en el tiempo respecta, a objetos externos, cuya realidad no se

${ }^{62} C R P$, p. 23 (nota 6 al prólogo de la segunda edición); $K r V$ B XXXIX.

${ }^{63} \mathrm{CRP}$, p. 135; KrV B 275-276. 
reduce a las representaciones del sujeto y que se hayan sometidos a o determinados temporalmente por un tiempo continuo-homogéneo real o natural que no es producido,sino representado por los principios del entendimiento puro, y de cuya realidad la efectiva y constante transformación natural da cuenta. De allí que, a mi parecer, no pueda decirse que Kant haya sido refutado por Husserl, en la medida en que el carácter continuo sucesivo del tiempo de la naturaleza sigue estando presupuesto por la propia descripcióm husserliana de la conciencia inmanente del tiempo. Consecuencia necesaria de esta afirmación precedente es que no es posible hacer visible al tiempo en sí mismo. En efecto, al no ser posible una fenomenología del tiempo como tiempo puramente conciencial, puesto que este tiempo presupone y está referido al tiempo como continuo homogéneo real, $y$ al captarse este tiempo real por las relaciones de los objetos y no en sí, hay que concluir que es tan necesario presuponer un tiempo real como imposile percibirlo independientemente de los objetos en el tiempo. Toda teoría del tiempo es una teoría indirecta. He aquí un primer límite a cualquier investigación sobre el tiempo. El tiempo en sí es irrepresentable. Haber visto con claridad este límite es quizás el legado imperecedero de Kant.

Pero, en segundo lugar, si bien es cierto que todo acto de conciencia está ligado, en lo que a su determinación temporal respecta, a objetos externos (en última instancia a la regularidad de los movimientos astrales), lo inverso también es cierto: toda determinación temporal de objetos externos, toda puesta en acto de los principios del entendimiento puro, implica un acto de autoconciencia. Por eso mismo el punto de partida de toda temporalización, incluso de la temporalización trascendental-objetiva del tiempo real es el ahora viviente de la conciencia y sus dimensiones de pasado y futuro. Haber visto con claridad este origen último es quizás el legado imperecedero de Husserl. La determinación trascendental de un tiempo realnatural en sí mismo inasible y su temporalización por la conciencia subjetiva no se oponen, sino que se imbrican y se presuponen mutuamente. Esta imbricación mutua no es sino la expresión concreta de la primera aporía de la temporalidad que constituye la última frontera a toda investigación sobre el tiempo: no hay intuición del tiempo sino en cuanto temporalizado por una conciencia, pero toda conciencia pasa en el tiempo.

Una última reflexión sobre la tesis tal vez más provocativa y -soy consciente de ello- más difícil de aceptarde este ensayo: presuponer en Kant una dimensión real del tiempo, aun cuando el filósofo una y otra vez nos 
dice que el tiempo en sí mismo no es nada y que constituye tan sólo una forma a priori de la intuición. ¿No representa esta teoría trascendental del tiempo el ápice de una filosofía idealista que saca el tiempo del afuera cósmico para ponerlo en el interior del sujeto? A esta pregunta responderé con otra: ¿no se reduce acaso el sentido, esto es, aquello sobre la base de lo cual se comprende la determinación trascendental del tiempo en Kant a la representación objetiva del tiempo de la naturaleza? Si esto es así, entonces, el sentido de la determinación trascendental y de la consecuente re-presentación ${ }^{64}$ objetiva del tiempo es, precisamente, el tiempo de la naturaleza. En todo esto artículo hemos considerado la teoría trascendental del tiempo justamente como una re-presentación objetiva y no como una producción o presentación subjetiva. Si bien esta tesis puede contradecir la letra de la Crítica, no contradice, estimo, su espíritu, en cuanto el sujeto kantiano se agota en representar la objetividad natural. Que a la idealidad trascendental del tiempo (y del espacio) corresponde, a modo de presupuesto, su realidad empírica es un hecho. Es el hecho que estudian y articulan las ciencias que se refieren al tiempo, ante todo la física. Es el hecho que testimonia la transformación del universo y las huellas que esa transformación deja y que la ciencia estudia. Es el hecho que testimonia bruta y crudamente nuestro envejecimiento. Por ello tiene razón Ricoeur cuando nos advierte que "la inherencia originaria del tiempo y del espacio respecto del sujeto, proclamada por la estética trascendental, no podría, (...), enmascarar la otra cara del problema e impedir plantear la pregunta: ¿qué tipo de realidad empírica corresponde a la idealidad trascendental?" 65 . Se trata de una realidad empírica inasible en sí, pero cuya representación objetiva, es decir, la representación de cómo se determinan las relaciones en el tiempo entre los objetos, es el tema de la analítica de los principios. De ello resulta que el tiempo, a pesar de ser representado subjetivamente, es el tiempo de una naturaleza, cuya objetividad está garantizada precisamente por esa representación, obra de un conjunto de principios trascendentales.

Ciertamente la teoría del tiempo en Kant -lo hemos visto- no ha ido más allá del marco irrebasable de la aporía. Pero ha tenido la grandeza de

${ }^{64}$ Justamente porque se trata de una re-presentación, de un traer al presente de la conciencia subjetiva, la re-presentación objetiva implica o encubre una fenomenología del tiempo conciencial.

65 TN III, p. 716. 
articularla, pues, en vez de tratar de reducir unívocamente el tiempo del cosmos al tiempo del sujeto o el tiempo del sujeto al del cosmos, Kant ha sabido ver en el tiempo el punto justo en que se cruzan el sujeto y el cosmos.

\title{
Resumen
}

Se analiza la estética trascendental y las analogías de la experiencia en la Crítica de la razón pura. Contra la afirmación kantiana del tiempo como mera forma de la intuición pura, el análisis procura mostrar: 1) en qué medida el tiempo real, cósmico o tiempo propiamente dicho, aun cuando no pueda ser asido en sí mismo, debe suponerse como determinante de las características generales de la temporalidad propia de las relaciones objetivas entre los fenómenos; y, 2) en qué medida esta temporalidad implica a su vez una fenomenología encubierta de la temporalidad conciencial inmanente.

Palabras clave: "Kant", "tiempo", "temporalidad"

\begin{abstract}
The article analyses the trascendental aesthetics and the analogies of experience in the Critique of Pure Reason. Against the kantian assertion about time as a mere form of pure intuition, the analysis endeavours to show: first, up to which extent real, cosmic time o time proper, even when it cannot be apprehended in itself, must be presupposed as determining the general characteristics of the temporality inhering in the objective relations among phenomena; and second: up to which extent does that temporality imply a covert phenomenology of the immanent conciential temporality.

Key words: "Kant", "time", "temporality"
\end{abstract}

